\title{
An Image Dehazing Method Based On an Improved Retinex Theory
}

\author{
Ebtesam Mohameed Alharbi ${ }^{1,2, a}$, Yilin Shan ${ }^{2, b}$, Peng Ge ${ }^{2,3, c}$, Hong Wang 1, 2, d,* \\ ${ }^{1}$ School of Electronics and Information, South China University of Technology, Guangzhou 510640, \\ China;
}

2Engineering Research Center for Optoelectronics of Guangdong Province, School of Physics and Optoelectronics, South China University of Technology, Guangzhou 510640, China;

3Institute of Electronic and Information, Hangzhou Dianzi University, Hangzhou310018, China.

a491569590@qq.com, b349736392@qq.com, cscpge@scut.edu.cn, dphhwang@scut.edu.cn

\begin{abstract}
Image quality is often affected in many ways by the atmosphere, especially in foggy weather conditions. Dehazing is a highly demanded operation within the domain of image processing for various applications. The paper proposes a new single-image dehazing algorithm based on the single scale retinex (SSR) algorithm, combined with the theory of atmospheric scattering. Compared with the SSR algorithm, the proposed algorithm is shown to effectively take advantage of the abundance of information within an image and offers a clearer dehazing output within distant image scenes. Compared with the histogram equalization algorithm, our algorithm achieves a higher degree of natural colour restoration and hence yields a high color fidelity. Additionally, the algorithm exhibits a higher efficiency in recovering image features from objective and subjective points of view.
\end{abstract}

Keywords: Image processing; Image reconstruction-restoration; Dehazing; Retinex theory.

\section{Introduction}

Imaging devices are often affected by the scattering, refraction and absorption of the suspended particles within the atmosphere in foggy weather conditions. In the first category are enhancement-based algorithms. This phenomenon leads to a significant degradation of the image acquired within such adverse conditions. The end result is the failure or sub-optimal performance of outdoor imaging systems within adverse conditions, a situation with serious implications for machine vision and image understanding systems. This motivates the work in this paper which aims to address the problem of haze image degradation towards an improvement of the performances of optical image processing and machine vision systems.

Presently, single image dehazing schemes fall into two main categories. These algorithms do not consider the physical model of the imaging principle but rather improve the image quality in order to satisfy requirements by blindly enhancing the contrast of the images and highlighting image details. Algorithms in this category include histogram equalization [1], retinex theory [2], and saliency extraction [3-6]. The second category comprises algorithms that are based on image recovery [7-15]. These algorithms are based on the physical model of atmospheric scattering and attempt to recover the image by means of some auxiliary or prior information. He's dark channel prior algorithm [10] is the most famous within this category although the complexity of the algorithm significantly impedes its implementation.

Drawing from the characteristics of color consistency [2], the retinex theory is very effective towards image enhancement in situations where the illumination of the imaging field is not sufficient or is not uniform. However, when the retinex theory is applied directly to image dehazing, the resulting dehazing effect is far from ideal. This paper proposes a new single frame dehazing algorithm based on the single scale retinex (SSR) algorithm [16], combined with the theory of atmospheric scattering. 


\section{Single scale retinex theory (SSR)}

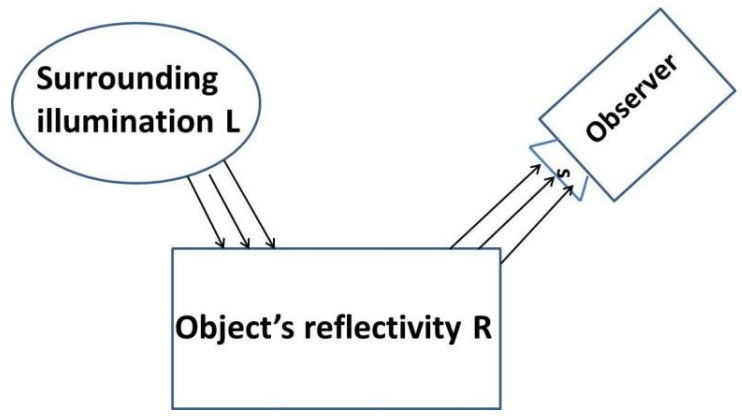

Fig. 1. The retinex theory

The retinex theory, originally proposed by Land and et al. presents a means of adjusting the color and intensity sensed by the viewer. The Fig. 1 lays out the fundamental principle of the retinex theory. It is clear that the intensity of the rays perceived by the viewer is formed through a combination of the surrounding illumination $\mathrm{L}$ and reflectance of the object. This expression is illustrated as follows:

$I(x, y)=L(x, y) \cdot R(x, y)$,

in the Eq. (1), $I(x, y)$ represents the perceived image intensity, $L(x, y)$ expresses the surrounding illumination, $R(x, y)$ expresses the reflectance of the object and $L(x, y)$ specifies the dynamic range of the image and it varies as the surrounding changes. The $\mathrm{R}(\mathrm{x}, \mathrm{y})$ component is the inwardness of the object. The basic idea of the retinex theory is to remove the surrounding illumination intensity $L(x, y)$ from the image $\mathrm{I}(\mathrm{x}, \mathrm{y})$ in order to obtain a resulting clear image. By applying the logarithm to equation (1), the reflectance $\mathrm{R}(\mathrm{x}, \mathrm{y})$ derived as:

$R(x, y)=\log (\mathrm{I}(\mathrm{x}, \mathrm{y}))-\log (\mathrm{L}(\mathrm{x}, \mathrm{y}))$.

In the single scale retinex theory (SSR), the surrounding illumination can be obtained through implementation with a Gaussian filter;

$L(x, y)=F(\mathrm{x}, y) * I(x, \mathrm{y})$,

In which $\mathrm{F}(\mathrm{x}, \mathrm{y})$ is the low-pass filter function, which is generally adopted as a Gaussian function, and $*$ denotes the convolution operator. $F(x, y)$ needs to satisfy the condition shown as below;

$$
F(x, y)=k e^{-\frac{r^{2}}{c^{2}}},
$$

In which $\mathrm{c}$ is the scale of the Gaussian function. It determines the degree of blurring. $\boldsymbol{k}$ is the normalized factor. Therefore, the single scale retinex theory is:

$R(x, y)=\log (I(x, y))-\log (F(x, y) * I(x, y))$.

The parameter $\mathrm{c}$ is the main factor affecting the single scale retinex theory. When $\mathrm{c}$ is small, the detail will be enhanced, the dynamic range compression will be better, but it will be easier to cause colour distortion. When $\mathrm{c}$ is large, the colour sensation will be better, but the detail will be fuzzier. The value of $\mathrm{c}$ is therefore vital to the performance of the algorithm.

\section{The atmospheric scattering model}

The atmospheric scattering model refers to the total radiation that imaging devices receive includes the radiation of the attenuated incident light [17] and the radiation of the atmospheric imaging system [18], that is;

$I(x)=I_{\infty} \rho e^{-\beta d}+I_{\infty}\left(1-e^{-\beta d}\right)$,

In which $\boldsymbol{I}_{\infty}$ represents the intensity of the environmental light, and $\rho$ is the reflectance of the scene. $\beta$ is the scattering coefficient and $d$ represents the depth of field. Additionally, $I_{\infty} \rho$ represents the radiation of the scene in the absence of fog. $e^{-\beta d}$ represents the transitivity of the scene. This represents the un-attenuated portion of the incident light which passes through the atmosphere and reaches to the imaging device. With an increase in the depth of the field $\mathrm{d}, e^{-\beta d}$ becomes smaller. When $e^{-\beta d}$ is smaller, it indicates that the attenuated portion is much higher. $I_{\infty} \rho e^{-\beta d}$ represents the attenuated portion of the incident light. $I_{\infty}\left(1-e^{-\beta d}\right)$ illustrates the impact 
that the environmental illumination has on the imaging processing. We can see that when the depth of the field increases, the scene radiation attenuates more significantly, and drops sharply in the imaging process, additionally, the portion of the environmental radiation in the imaging process becomes much larger. This results in the low contrast of the far scenes within the image and the image demonstrates white patches within these regions. From equation (6) it can be seen that a haze free image can be obtained by removing the effect of $I_{\infty}\left(1-e^{-\beta d}\right)$ and $e^{-\beta d}$.This is the core of our improved algorithm.

\section{An improved defogging method based on single-scale retinex theory}

When dehazing is performed using the previous single scale retinex theory (SSR), the effect caused by the atmosphere scattering is actually regarded as the radiation part $\mathrm{L}(\mathrm{x})$ in the retinex theory. However, according to the atmospheric scattering model, there is not only a multiplicative component to the radiation $\mathrm{L}(\mathrm{x})$, but also an additive component. This may induce some estimation errors. This can mathematically be expressed as:

$$
R(x)=\frac{I(x)}{L(x)}=\frac{I_{\infty} \rho e^{-\beta d}+I_{\infty}\left(1-e^{-\beta d}\right)}{L(x)} .
$$

The radiation estimation is an estimation process of a multiplicative component. In the atmospheric scattering model, we can regard the atmospheric imaging component an addictive component. So we can approximately estimate the illumination component as $\boldsymbol{e}^{-\boldsymbol{\beta} \boldsymbol{d}}$, which is illustrated as:

$L(\mathrm{x})=\mathrm{e}^{-\beta d}$.

By substituting into the equation (7), we derive:

$$
R(x)=I_{\infty} \rho+I_{\infty}\left(\frac{1}{e^{-\beta d}}-1\right) \text {. }
$$

From the above, we can see that through single scale processing, there is a $\boldsymbol{I}_{\infty}\left(\frac{\mathbf{1}}{\boldsymbol{e}^{-\beta d}}-\mathbf{1}\right)$ component besides $\boldsymbol{I}_{\infty} \boldsymbol{\rho}$. Therefore with an increase in the depth of the field, the effect caused by the second component in equation (9) will be more significant. This is the reason why the removal of haze in distant scenes of the image is much more challenging.

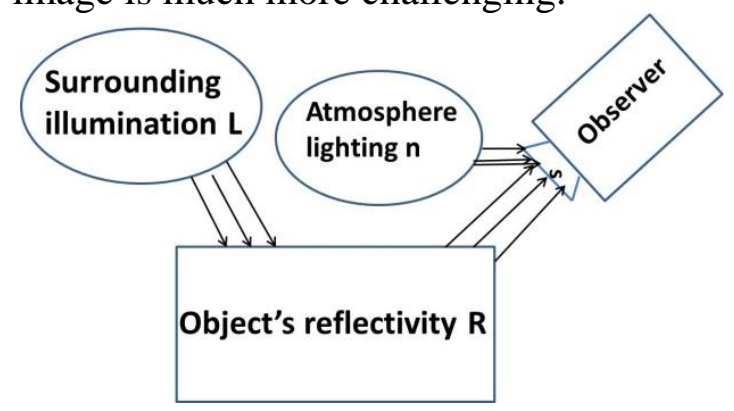

Fig. 2 The retinex theory with a foggy weather

So here we propose a new retinex algorithm as shown in Fig.2. In the foggy weather conditions, the colour and the brightness perceived are not only decided by the environmental illumination and object reflectance, but also is affected by the atmospheric lighting $\mathrm{n}$. The new expression therefore is;

$$
I(x, y)=R(x, \mathrm{y}) \mathrm{L}(\mathrm{x}, \mathrm{y})+\mathrm{n}(\mathrm{x}, \mathrm{y}),
$$

In which $n(x, y)$ is the component that is directly contributed by the atmospheric light during the imaging process. This additive component can be estimated by means of a low-pass filter and efficiently removed from the image. This allows for the original form of the equation (1) to be obtained. The flow chart of the algorithm is shown in Fig. 3 and the basic steps of the algorithm is:

(1) We estimate $n(x, y)$ by a Gaussian function filter, and remove it from the original image.

(2) Normalize the result of (1) and obtain the intermediate result.

(3) We estimate $L(x, y)$ by a Gaussian function filter and take the logarithm of the result.

(4) We derive automatic color order processing of the above result in order to eliminate the overexposure phenomenon. 
(5) Obtain the final dehazing results.

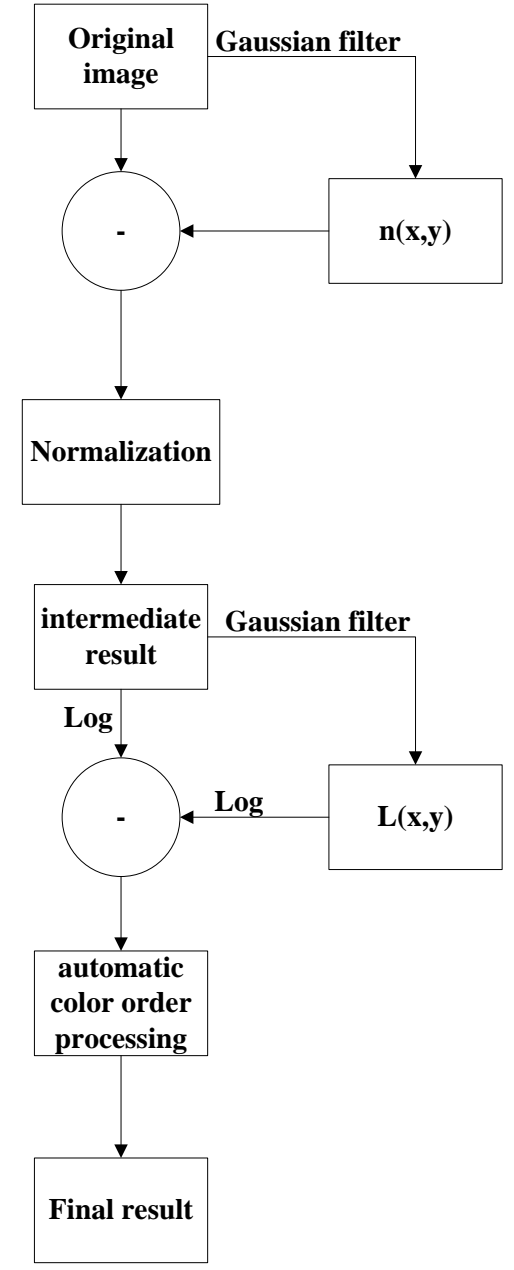

Fig. 3 The flow chart of the new algorithm

\section{Experimental results}

In the experiments we select the scale parameter in the Gaussian function $\mathrm{c}=80$, and follow the steps (1) and (3). Fig.4 presents our obtained results and its comparison to the original SSR algorithm. The automatic colour order processing of the SSR algorithm is obtained. It is observed that the scene within the distant fields of the image are much clearer and the detail is more pronounced through implementation with the proposed algorithm.

Five methods are used to assess the dehazing effect, including information entropy, average gradient, edge strength, variance and colour reducibility [19]. The dehazing effect attained is significantly better if the data in the first four columns in table 1 are larger. This shows that the image is much clearer and the detail is more plentiful. Furthermore, the colour reducibility in column 5 indicates the color distortion of the algorithm. If this data is bigger, then the distortion will be less, resulting in a better dehazing result. From the data in table 1, we can see that our improved algorithm surpasses the SSR algorithm in all aspects. It greatly improves the dehazing effect.

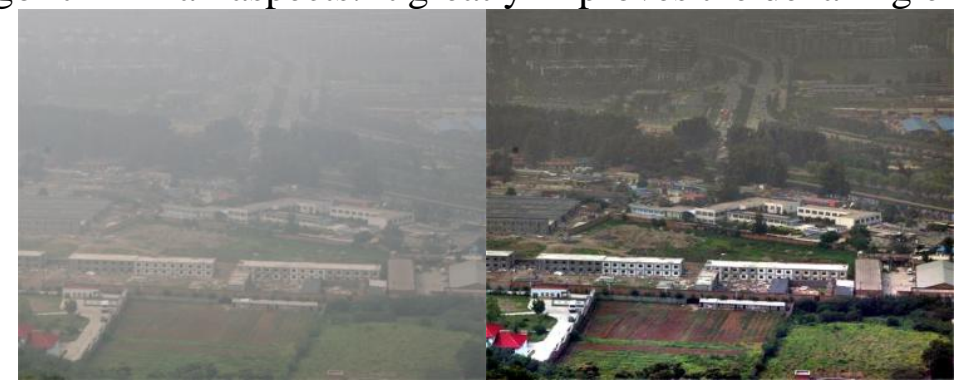

(a)

(b) 


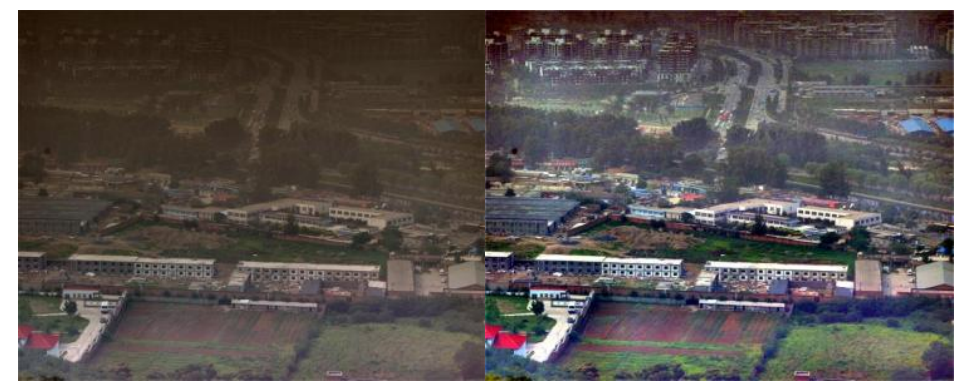

(c)

(d)

Fig. 4 experimental results;(a) Original image;(b) processed by SSR algorithm;(c)intermediate result by our improved algorithm;(d)final result by our improved algorithm.

Table 1 Objective assessment of the dehazing image

\begin{tabular}{c|c|c|c|c|c}
\hline & $\begin{array}{c}\text { information } \\
\text { entropy }\end{array}$ & average gradient & edge strength & Variance & color reducibility \\
\hline Original image & 13.3175 & 3.0172 & 20.9402 & 492.6031 & - \\
SSR & 14.2545 & 10.3790 & 73.4939 & 1489.5 & 0.6366 \\
Improved & 15.2136 & 13.7727 & 97.7731 & 1981.7 & 0.6589 \\
algorithm & 1969 \\
\hline
\end{tabular}

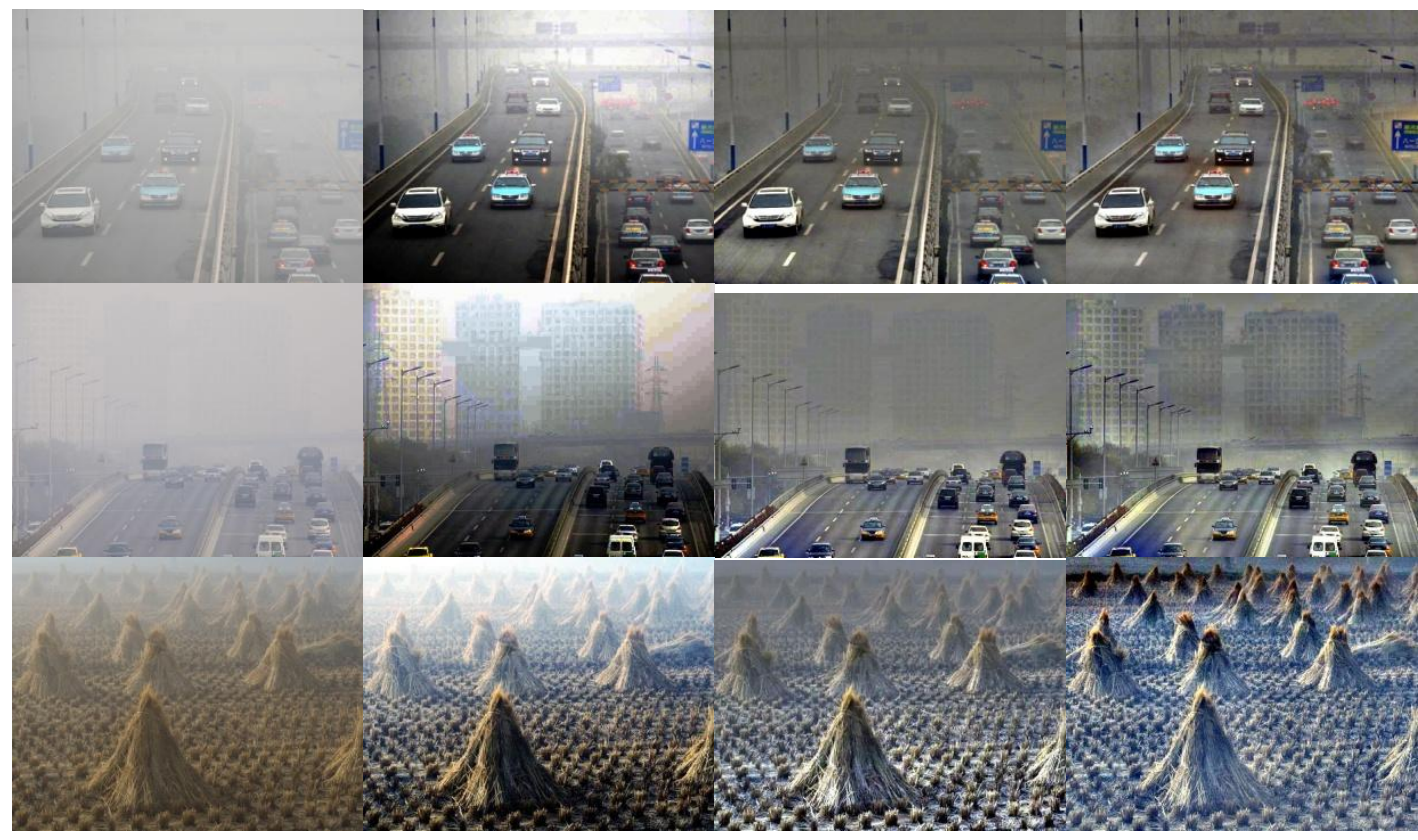

(a)

(b)

(c)

(d)

Fig. 5 (a) Original image; (b) Processed by histogram equalization; (c) processed by SSR; (d) Our method.

Fig. 5 presents additional results and a comparison with histogram equalization and the original SSR algorithm. We can see that compared with SSR algorithm, our algorithm achieves results with a higher degree of detail and with higher image quality in the far fields of the scene. Compared with histogram equalization, our algorithm attains a much holistic natural colour effect, and hence attains a better color fidelity. Additionally the features in the far fields of the scene are more enhanced and abundant.

\section{Conclusion}

The paper proposes a dehazing algorithm based on an improved retinex theory. Compared with SSR algorithm and histogram equalization algorithm, our algorithm shows a significant advantage in recovering the details and the colour from both the objective and subjective assessments. 
Although our improved algorithm shows more effective performance in dehazing, some drawbacks still remain. In some images with large sky portions, A little variation is derived in the dehazing result. By removing $\mathrm{n}(\mathrm{x}, \mathrm{y})$, we may also remove the whole sky and this may result a darkening of the sky regions. Additionally, some colour distortions still remain, a phenomenon illustrated in the Fig. 5. Tackling these drawbacks is the focus of future extensions of this paper.

\section{Acknowledgements}

This work was supported by the Key technologies R\&D Program of Guangdong Province (2014B010119002), the Natural Science Foundation of Guangdong Province (Nos.2015A030310278, 2016A030313473), and partly by Zhejiang Key Discipline of Instrument Science and technology (No. JL150510), and Supported by Hunan Province Key Laboratory of Videometric and Vision Navigation.

\section{References}

[1]. J. H. Kim, J. Y. Sim, and C. S. Kim.Single Image Dehazing Based on Contrast Enhancement. ICASSP, 2011, p.1273-1276.

[2]. Land E H, Mccann J J.Lightness and retinex theory. Journal of Optical Society of America, 61(1971),p.1-11.

[3]. Zhao J, Chen Y, Feng H, et al, Infrared image enhancement through saliency feature analysis based on multi-scale decomposition [J]. Infrared Physics \& Technology, 62(2014),p. 86-93.

[4]. Zhao J, Gao X, Chen Y, et al. Fast iterative adaptive nonuniformity correction with gradient minimization for infrared focal plane arrays. Infrared Physics \& Technology,65(2014),p. 87-93.

[5]. Zhao J, Chen Y, Feng H, et al.Fast image enhancement using multi-scale saliency extraction in infrared imagery. Optik-International Journal for Light and Electron Optics,125(2014),p.40394042.

[6]. Jufeng Zhao, Xiumin Gao, Yueting Chen, Huajun Feng. Optical imaging system-based real-time image saliency extraction method .Optical Engineering 54(2015),p. 043101.

[7]. R. Tan, Visibility in Bad Weather from a Single Image Proc. IEEE Conf. Computer Vision and Pattern Recognition, June 2008.

[8]. R. Fattal, Single image dehazing, ACM Transactions on Graphics, 27 (2008),p. 1-9.

[9]. Jean-Philippe T, Nicolas H. Fast visibility restoration from a single color or gray level image[C]//Proceeding of IEEE 12th International Conference on Computer Vision New York, USA;IEEE,2009;2201-2208.

[10]. K. M.He, J. Sun, and X. O. Tang.Single image haze removal using dark channel prior.IEEE Trans. Pattern Anal. Mach. Intell. 33(2010), p.2341-2353.

[11]. S. Y. Tao, H. J. Feng, Z. H. Xu, Q. Li, Image degradation and recovery based on multiple scattering in remote sensing and bad weather condition, Optics Express, 20(2012),p. 16584-16595.

[12]. S. Fang, X. Xia, X. Huo, and C. Chen.Image dehazing using polarization effects of objects and airlight. Opt. Express 22(2014), p.19523-19537.

[13]. F. Liu, L. Cao, X. Shao, P. Han, and X. Bin.Polarimetric dehazing utilizing spatial frequency segregation of images.Appl. Opt. 54(2015), p.8116-8122.

[14]. C. Yeh, L. Kang, M. Lee, and C. Lin.Haze effect removal from image via haze density estimation in optical model.Opt. Express 21(2013), p.27127-27141. 
[15]. J. Liang, L. Ren, E. Qu, B. Hu, and Y. Wang.Method for enhancing visibility of hazy images based on polarimetric imaging.Photon. Res. 2(2014), p.38-44.

[16]. Jobson D J, Rrahman Z, Woodell G A. Properties and performance of a center/surround retinex. IEEE Trans. Image Processing, 6(1997),p.451-462

[17]. Narasimhan S G, Nayar S K. Shedding light on the weather [C], Computer Vision

[18]. and Pattern Recognition, 1(2003),p. 665-672.

[19]. Narasimhan S G, Nayar S K. Vision and the atmosphere. International Journal of Computer Vision, 48(2002),p. 233-54.

[20]. Li Dapeng, Yu Jing, Xiao Chuangbai. No-reference quality assessment method for defogged images. Journal of Image and Graphics, 16(2011),p. 1753-1757. 